\title{
A remark on prime ideals
}

\author{
S.C. LEE ${ }^{1}$, R. VARMAZYAR ${ }^{2, @}$ \\ ${ }^{1}$ Department of Mathematics Education and Institute of Pure and Applied Mathematics \\ Jeonbuk National University, Jeonju, Jeonbuk 54896, South Korea \\ ${ }^{2}$ Department of Mathematics, Khoy Branch, Islamic Azad University \\ Khoy 58168-44799, Iran \\ scl@jbnu.ac.kr, varmazyar@iaukhoy.ac.ir
}

Received September 25, 2019

Presented by Consuelo Martínez

Accepted January 14, 2020

Abstract: If $M$ is a torsion-free module over an integral domain, then we show that for each submodule $N$ of $M$ the envelope $E_{M}(N)$ of $N$ in $M$ is an essential extension of $N$. In particular, if $N$ is divisible then $E_{M}(N)=N$. The last condition says that $N$ is a semiprime submodule of $M$ if $N$ is proper.

Let $M$ be a module over a ring $R$ such that for any ideals $\mathfrak{a}, \mathfrak{b}$ of $R,(\mathfrak{a} \cap \mathfrak{b}) M=\mathfrak{a} M \cap \mathfrak{b} M$. If $N$ is an irreducible and weakly semiprime submodule of $M$, then we prove that $\left(N:_{R} M\right)$ is a prime ideal of $R$. As a result, we obtain that if $\mathfrak{p}$ is an irreducible ideal of a ring $R$ such that $\mathfrak{a}^{2} \subseteq \mathfrak{p}$ (a is an ideal of $R) \Rightarrow \mathfrak{a} \subseteq \mathfrak{p}$, then $\mathfrak{p}$ is a prime ideal.

Key words: Prime ideal; generalized prime submodule; semiprime submodule; weakly semiprime submodule.

AMS Subject Class. (2010): 13C05, 18E40, 13B30, 16D60, $13 \mathrm{~B} 25$.

\section{INTRODUCTION AND PRELIMINARIES}

Throughout this paper, all rings are commutative with identity and all modules are unitary.

For definitions, examples, of prime, weakly prime, semiprime, and weakly semiprime submodules of $R$-modules, and relations among them we refer the reader to $[8]$. In this paper, we do not deal with weakly prime, but we deal with prime, semiprime, and weakly semiprime submodules of $R$-modules. More precisely, let's consider the three conditions, for a submodule $N$ of an $R$ module $M$, given as follows:

1. For $a \in R, m \in M$ with $a m \in N$, either $a \in\left(N:_{R} M\right)$ or $m \in N$.

2. For each $a \in R, m \in M$ with $a^{2} m \in N$, am $\in N$.

3. For each $a \in R$ with $a^{2} M \subseteq N, a M \subseteq N$.

@ Corresponding author

ISSN: 0213-8743 (print), 2605-5686 (online) 
A proper submodule $N$ of an $R$-module $M$ is said to be prime, semiprime, and weakly semiprime if it satisfies the condition (1), the condition (2), and the condition (3), respectively.

We can summarize the results as follows:

$$
\text { prime } \underset{k}{\Rightarrow} \text { semiprime } \underset{\forall}{\Rightarrow} \text { weakly semiprime }
$$

Moreover, if $N$ is a prime submodule of an $R$-module $M$ then it follows from [11, Proposition 2.4] that $(N: M)$ is a prime ideal of $R$. By [11, Proposition 2.4], every prime submodule is semiprime and by [4, Theorem 11] there are some semiprime submodules that are not prime. It was shown in 8 ] that every semiprime submodule is weakly semiprime and the converse is not true in general.

The concept of prime number in the ring of integers is generalized to the one of prime ideal of a ring. In turn, the concept of prime ideal of a ring is generalized to the one of prime submodule of a module.

Several authors have studied the subject for a couple of decades and many results have been given to explore the nature of prime ideals and related concepts.

Section 2 is devoted to primes and generalized primes. If $M$ is a finitely generated multiplication module over a ring $R$, then it is shown that for any submodule $N$ of $M, \operatorname{rad}_{M}(N)=\sqrt{\left(N:_{R} M\right)} M$ (Theorem 2.1). Let $R$ be a ring. Let $M$ be an $R$-module. Assume that $M$ is a multiplication $R$-module, and that for any ideals $\mathfrak{a}, \mathfrak{b}$ of $R,\left(P:_{M} \mathfrak{b}\right) \cap\left(P:_{M} \mathfrak{a}\right) \subseteq P$. Then we show that $P$ is a generalized prime submodule of an $R$-module $M$ if and only if $\left(P:_{R} M\right)$ is a prime ideal of $R$ (Theorem 2.2).

In Section 3, we concerned about semiprime submodules. Let $M$ be a torsion-free module, or a projective module over an integral domain $R$. Then we show that for every submodule $N$ of $M, E_{M}(N)$ is an essential extension of $N$, and in particular, for every divisible submodule $N$ of $M, E_{M}(N)=N$ and $E_{M}(N)$ is the injective envelope of $N$ (Theorem 3.1. Corollary 3.1).

In Section 4, we deal with weakly semiprimes. If $M$ is a module over a ring $R$ such that for any ideals $\mathfrak{a}, \mathfrak{b}$ of $R,(\mathfrak{a} \cap \mathfrak{b}) M=\mathfrak{a} M \cap \mathfrak{b} M$ and if $N$ is an irreducible and weakly semiprime submodule of $M$, then we prove that $\left(N:_{R} M\right)$ is a prime ideal of $R$ (Theorem 4.1). In particular, if $\mathfrak{p}$ is an irreducible ideal of a ring $R$ such that $\mathfrak{a}^{2} \subseteq \mathfrak{p}(\mathfrak{a}$ is an ideal of $R) \Rightarrow \mathfrak{a} \subseteq \mathfrak{p}$, then we show that $\mathfrak{p}$ is a prime ideal of $R$ (Corollary 4.2). 
Notations: Let $\operatorname{Spec}(M)$ be the spectrum of prime submodules of an $R$-module $M$. For a submodule $N$ of an $R$-module $M$ and an ideal $\mathfrak{a}$ of a ring $R$, we write

$$
\begin{aligned}
\left(N:_{R} M\right) & =\{a \in R: a M \subseteq N\}, \\
(N: M \mathfrak{a}) & =\{x \in M: r x \in N \text { for all } r \in \mathfrak{a}\}, \\
V(N) & =\{P \in \operatorname{Spec}(M): P \supseteq N\}, \\
\sqrt{\mathfrak{a}} & =\left\{a \in R: a^{n} \in \mathfrak{a} \text { for some positive integer } n\right\} .
\end{aligned}
$$

For undefined terminologies and notations, we refer the reader to references [10, 6].

\section{PRIMES AND GENERALIZED PRIMES}

This section is dedicated to the relationship between primes and generalized primes.

If $R$ is a ring and $\mathfrak{a}$ is an ideal of $R$, then it is well-known that the radical $\sqrt{\mathfrak{a}}$ of $\mathfrak{a}$ is an ideal of $R$ (see, for example, [2, p. 33]), and $\sqrt{\mathfrak{a}}=\cap_{\mathfrak{p} \in V(\mathfrak{a})} \mathfrak{p}$ (see, for example, [2, Corollary 2.12]).

Definition 2.1. Let $N$ be a submodule of an $R$-module $M$. Then the radical of $N$ in $M$ is defined to be the intersection of prime submodules of $M$ containing $N$, and is denoted by $\operatorname{rad}_{M}(N)$. That is, $\operatorname{rad}_{M}(N)=\cap_{P \in V(N)} P$.

Remark 2.1. For any $R$-module $M, \operatorname{rad}_{M}(M)=M$ because $V(M)=\emptyset$.

Definition 2.2. For any $R$-module $M$, the radical of $M$ is defined to be the radical of the zero submodule in $M$ and is denoted by $\operatorname{rad}(M)$. That is,

$$
\operatorname{rad}(M)=\operatorname{rad}_{M}(0)=\cap_{P \in \operatorname{Spec}(M)} P .
$$

An ideal $\mathfrak{a}$ of $R$ is called a radical ideal if $\mathfrak{a}=\sqrt{\mathfrak{a}}$ (see [2, p. 33]).

Definition 2.3. A submodule $N$ of an $R$-module $M$ is called a radical submodule of $M$ if $\operatorname{rad}_{M}(N)=N$.

Every module is the radical submodule of itself. An $R$-module $M$ is called a multiplication module if for each submodule $N$ of $M, N=\left(N:_{R} M\right) M$ (see [1, 3]). 
THEOREM 2.1. Let $M$ be a finitely generated multiplication module over a ring $R$. Then for any submodule $N$ of $M$,

$$
\begin{aligned}
\operatorname{rad}_{M}(N) & =\cap_{\mathfrak{p} \in V\left(N:_{R} M\right)}(\mathfrak{p} M) \\
& =\left(\cap_{\mathfrak{p} \in V\left(N:_{R} M\right)} \mathfrak{p}\right) M=\sqrt{\left(N:_{R} M\right)} M .
\end{aligned}
$$

Proof. A map $\varphi: \operatorname{Spec}\left(R /\left(N \quad:_{R} M\right)\right) \rightarrow \operatorname{Spec}(M / N)$ defined by $\varphi\left(\mathfrak{p} /\left(N:_{R} M\right)\right)=(\mathfrak{p} M) / N$ is an order-preserving 1:1 correspondence (see [3]), so that the first equality follows. The second one follows from the fact that $M$ is a multiplication module, and the third one follows from the fact in the first paragraph of this section.

Corollary 2.1. ([2, Corollary 2.12]) If $R$ is a ring and $\mathfrak{a}$ is an ideal of $R$, then $\sqrt{\mathfrak{a}}=\cap_{\mathfrak{p} \in V(\mathfrak{a})} \mathfrak{p}$.

Let $R$ be a ring, then every prime ideal of $R$ has the condition:

$$
\mathfrak{a} \cap \mathfrak{b} \subseteq \mathfrak{p}(\mathfrak{a}, \mathfrak{b} \text { are ideals of } R) \quad \Rightarrow \quad \mathfrak{a} \subseteq \mathfrak{p} \text { or } \mathfrak{b} \subseteq \mathfrak{p}
$$

However, a prime submodule $P$ of $M$ does not satisfy the following condition in general:

$$
A \cap B \subseteq P(A, B \text { are submodules of } M) \Rightarrow \text { either } A \subseteq P \text { or } B \subseteq P .
$$

An example of this is given below.

ExAmPle 2.1. Let $R$ be an integral domain. Then $R^{2}(=R \oplus R)$ is a torsion-free $R$-module, so that the submodule $\langle(0,0)\rangle$ is a prime submodule. Moreover, $(R \times\langle 0\rangle) \cap(\langle 0\rangle \times R)=\langle(0,0)\rangle$, but $R \times\langle 0\rangle \neq\langle(0,0)\rangle$, and $\langle 0\rangle \times R \neq\langle(0,0)\rangle$. This shows that the zero submodule of $R^{2}$ does not satisfies the condition (2.1).

A proper submodule $P$ of an $R$-module $M$ is called generalized prime if $P$ satisfies condition $(2.1$.

Lemma 2.1. Assume that $M$ is a multiplication $R$-module. If $P$ is a prime submodule of an $R$-module $M$, then $P$ is a generalized prime submodule of $M$. 
Proof. Assume that $A \cap B \subseteq P$. Let $\mathfrak{a}, \mathfrak{b}$ be ideals of $R$ such that $A=\mathfrak{a} M$ and $B=\mathfrak{b} M$. Then $(\mathfrak{a} \mathfrak{b}) M \subseteq \mathfrak{a} M \cap \mathfrak{b} M=A \cap B \subseteq P$, so that $\mathfrak{a} \mathfrak{b} \subseteq\left(P:_{R} M\right)$. Notice that $\left(P:_{R} M\right)$ is a prime ideal of $R$. Then either $\mathfrak{a} \subseteq\left(P:_{R} M\right)$ or $\mathfrak{b} \subseteq\left(P:_{R} M\right)$. Hence either $\mathfrak{a} M \subseteq P$ or $\mathfrak{b} M \subseteq P$, that is, either $A \subseteq P$ or $B \subseteq P$. Therefore, $P$ is generalized prime.

Corollary 2.2. Let $R$ be a ring. Then every prime ideal of $R$ is generalized prime.

Not every generalized prime ideal of a ring $R$ is prime. An example of this is given below.

Example 2.2. Let $p$ be a prime number. Consider the ring

$$
\mathbb{Z}_{p^{2}}=\left\{0,1,2, \ldots, p^{2}-1\right\} .
$$

Then its only ideals are $\langle 0\rangle,\langle p\rangle$, and $\mathbb{Z}_{p^{2}}$. Hence it is easy to see that the zero ideal $\langle 0\rangle$ is not prime, but generalized prime.

Lemma 2.2. Assume that for any ideals $\mathfrak{a}, \mathfrak{b}$ of $R$,

$$
\left(P:_{M} \mathfrak{b}\right) \cap\left(P:_{M} \mathfrak{a}\right) \subseteq P .
$$

If $P$ is a generalized prime submodule of an $R$-module $M$, then $\left(P:_{R} M\right)$ is a prime ideal of $R$.

Proof. Let $\mathfrak{a} \mathfrak{b} \subseteq\left(P:_{R} M\right)$. Then $(\mathfrak{a} \mathfrak{b}) M \subseteq P$, so that $\mathfrak{a} M \subseteq\left(P:_{M} \mathfrak{b}\right)$ and $\mathfrak{b} M \subseteq\left(P:_{M} \mathfrak{a}\right)$. By our hypothesis $\mathfrak{a} M \cap \mathfrak{b} M \subseteq P$. Since $P$ is generalized prime, either $\mathfrak{a} M \subseteq P$ or $\mathfrak{b} M \subseteq P$. So, either $\mathfrak{a} \subseteq\left(P:_{R} M\right)$ or $\mathfrak{b} \subseteq\left(P:_{R} M\right)$. Therefore $\left(P:_{R} M\right)$ is a prime ideal of $R$.

Corollary 2.3. Let $R$ be a ring. Assume that for any ideals $\mathfrak{a}, \mathfrak{b}$ of $R$,

$$
(\mathfrak{p}: R \mathfrak{b}) \cap(\mathfrak{p}: R \mathfrak{a}) \subseteq \mathfrak{p} .
$$

If $\mathfrak{p}$ is a generalized prime ideal of $R$, then $\mathfrak{p}$ is a prime ideal of $R$.

Let's summarize the results as follows:

Theorem 2.2. Let $R$ be a ring. Let $M$ be an $R$-module. Assume that $M$ is a multiplication $R$-module, and that for any ideals $\mathfrak{a}, \mathfrak{b}$ of $R$,

$$
\left(P:_{M} \mathfrak{b}\right) \cap\left(P:_{M} \mathfrak{a}\right) \subseteq P .
$$

Then $P$ is a generalized prime submodule of $M$ if and only if $\left(P:_{R} M\right)$ is a prime ideal of $R$. 
Proof. $(\Rightarrow)$ : This follows from Lemma 2.2 .

$(\Leftarrow)$ : This follows from [3, Corollary 2.11] and Lemma 2.1.

Corollary 2.4. Let $R$ be a ring. Assume that for any ideals $\mathfrak{a}, \mathfrak{b}$ of $R$,

$$
\left(\mathfrak{p}:_{R} \mathfrak{b}\right) \cap\left(\mathfrak{p}:_{R} \mathfrak{a}\right) \subseteq \mathfrak{p} .
$$

Then $\mathfrak{p}$ is a prime ideal of $R$ if and only if $\mathfrak{p}$ is a generalized prime ideal of $R$.

\section{SEmiprimes}

In this section, we consider what conditions can be given to an $R$-module $M$ and to its submodule $N$, in order for a submodule $N$ of an $R$-module $M$ to be a semiprime submodule.

Let $M$ be an $R$-module, and let $N$ be a submodule of $M$. Then recall that the envelope of $N$ in $M$ is defined by

$$
E_{M}(N):=\left\langle\cup_{r \in R}\left(r M \cap\left(N:_{M} r\right)\right)\right\rangle=\left\langle G_{M}(N)\right\rangle
$$

(see [8, p. 3744]).

For every submodule $N$ of an $R$-module $M, N \subseteq E_{M}(N) \subseteq M$. In particular, $E_{M}(M)=M$ for every $R$-module $M$.

Lemma 3.1. For every proper submodule $N$ of an $R$-module $M$, the following assertions are equivalent:

(a) $E_{M}(N)=N$.

(b) $N$ is semiprime.

(c) For each $r \in R, m \in M$ with $r^{k} m \in N$ for some positive integer $k$, $r m \in N$.

Proof. $(a) \Rightarrow(b)$ : Let $r \in R, m \in M$ with $r^{2} m \in N$. Then $r m \in$ $r M \cap\left(N:_{R} r\right) \subseteq \cup_{a \in R}\left(a M \cap\left(N:_{R} a\right)\right) \subseteq E_{M}(N)=N$.

$(b) \Rightarrow(a)$ : To show that $E_{M}(N)=N$, it suffices to prove that $\cup_{r \in R}(r M \cap$ $\left.\left(N:_{M} r\right)\right) \subseteq N$. Let $x \in \cup_{r \in R}\left(r M \cap\left(N:_{M} r\right)\right)$. Then there exist $r \in R$, $m \in M$ such that $x=r m$ and $x \in\left(N:_{R} r\right)$. So, $r^{2} m=r x \in N$. By assumption, $x=r m \in N$.

(b) $\Rightarrow(c)$ : Let $r \in R$ and $m \in M$ with $r^{k} m \in N$ for some positive integer $k$. If $k \geq 3$, then $r^{2}\left(r^{k-2} m\right)=r^{k} m \in N$; hence by $(b) r^{k-1} m=r\left(r^{k-2} m\right) \in N$. By proceeding this way, we can get $r^{2} m \in N$. Hence by (b) again we get $r m \in N$.

$(c) \Rightarrow(b)$ is obvious. 
Example 3.1. Consider the ring $\mathbb{Z}$ of integers. Let $n>1$. Then $n \mathbb{Z}$ is a semiprime ideal of $\mathbb{Z}$ if and only if $n$ is a product of distinct prime numbers. For example, $12 \mathbb{Z}$ is not semiprime (for $2^{2} \cdot 3 \in 12 \mathbb{Z}$, but $2 \cdot 3 \notin 12 \mathbb{Z}$ ), but $6 \mathbb{Z}$ is semiprime.

If $E$ is divisible, then not every submodule of $E$ is divisible. For example $\mathbb{Q}$ is divisible as a $\mathbb{Z}$-module but its submodule $\mathbb{Z}$ is not.

If $M$ is a torsion-free module over an integral domain $R$, then every submodule of $M$ is also torsion-free.

It is well-known [10] that every $R$-module $M$ has an injective envelope $E(M)$, but not necessarily unique. (see [12] for examples).

It is fairly well-known that every nonsingular module $M$ over $R$ has the unique injective envelope $E(M)$ (see [7, Lemma 2]). Every torsion-free module $M$ over an integral domain $R$ is nonsingular, so that its injective envelope $E(M)$ is unique.

Theorem 3.1. Let $M$ be a torsion-free module over an integral domain $R$. Then the following statements are true:

(1) For every submodule $N$ of $M, E_{M}(N)$ is an essential extension of $N$.

(2) For every divisible submodule $N$ of $M, E_{M}(N)=N$ and $E_{M}(N)$ is the injective envelope of $N$.

Proof. (1) Let $N$ be any submodule of $M$, and let $U$ be a nonzero submodule of $E_{M}(N)$. We can take a nonzero element $x \in U$. Then there are $a_{1}, \ldots, a_{n} \in R$ and $x_{1}, \ldots, x_{n} \in G_{M}(N)$ such that $x=a_{1} x_{1}+\cdots+a_{n} x_{n}$. We may assume that all $x_{i}$ 's are nonzero. For each $i \in \mathbb{Z}_{n}$, there is $r_{i} \in R$ such that $x_{i} \in r_{i} M \cap\left(N:_{M} r_{i}\right)$. Moreover, all $r_{i}$ 's are nonzero and $r_{i} x_{i} \in N$. Since $M$ is torsion-free over an integral domain $R,\left(r_{1} \cdots r_{n}\right) x \neq 0$ and

$$
\left(r_{1} \cdots r_{n}\right) x=\left(a_{1} r_{2} \cdots r_{n}\right)\left(r_{1} x_{1}\right)+\cdots+\left(a_{n} r_{1} \cdots r_{n-1}\right)\left(r_{n} x_{n}\right) \in U \cap N .
$$

Hence $N$ is essential in $E_{M}(N)$.

(2) Let $N$ be any divisible submodule of $M$. Then $N$ is torsion-free divisible. Hence it follows from [10, Proposition 2.7] that $N$ is injective. Hence by (1) and [10, Theorem 2.17], $E_{M}(N)=N$. Moreover, notice that $E_{M}(N)$ is also injective. Then by (1) $E_{M}(N)$ is the injective envelope of $N$.

In Theorem 3.1 (2), the condition "divisible" cannot be deleted. To see this let $R=\mathbb{Z}, M=\mathbb{Q}$, and $N=\mathbb{Z}$. Then it is not difficult to see that $E_{M}(N)=\mathbb{Q} \neq N$. 
By [5, Exercise 9G, Section 9, Chapter I], every projective module over an integral domain $R$ is torsion-free.

Corollary 3.1. Let $M$ be a projective module over an integral domain $R$. Then (1) and (2) in Theorem 3.1 hold.

Compare the following Corollary $3.2(3)$ with [10, Theorem 2.8].

COROLlary 3.2. Let $R$ be an integral domain. Then the following assertions are true:

(1) Every torsion-free divisible $R$-module is injective ([10, Proposition 2.7]).

(2) For every ideal $\mathfrak{a}$ of $R, E_{R}(\mathfrak{a})$ is an essential extension of $\mathfrak{a}$.

(3) Let $\mathfrak{a}$ be an ideal of $R$. Then $\mathfrak{a}$ is injective if and only if it is divisible.

Corollary 3.3. Let $M$ be a torsion free module over an integral domain $R$. Then every injective submodule $N$ of $M$ is semiprime and $E_{M}(N)$ is the injective envelope of $N$.

Proof. By [10, Proposition 2.6], $N$ is divisible.

Corollary 3.4. Let $R$ be an integral domain. Then every divisible ideal of $R$ is semiprime and injective.

\section{WEAKLY SEMIPRIMES}

In this section, we will consider weakly semiprimes.

Lemma 4.1. For each proper submodule $N$ of an $R$-module $M$ the following assertions are equivalent:

(a) $N$ is weakly semiprime.

(b) For each ideal $\mathfrak{a}$ of $R$ with $\mathfrak{a}^{2} M \subseteq N, \mathfrak{a} M \subseteq N$.

(c) For each ideal $\mathfrak{a}$ of $R$ with $\mathfrak{a}^{2} \subseteq\left(N:_{R} M\right), \mathfrak{a} \subseteq\left(N:_{R} M\right)$.

(d) $\left(N:_{R} M\right)$ is a semiprime ideal of $R$.

Proof. $\quad(a) \Rightarrow(b)$ : Let $N$ be any weakly semiprime submodule of $M$, and let $\mathfrak{a}$ be any ideal of $R$ with $\mathfrak{a}^{2} M \subseteq N$. Let $x \in \mathfrak{a} M$. Then there exist $a_{1}, \ldots, a_{n} \in \mathfrak{a}$ and $x_{1}, \ldots, x_{n} \in M$ such that $x=a_{1} x_{1}+\cdots+a_{n} x_{n}$. For 
each $i \in \mathbb{Z}_{n}, a_{i}^{2} M \subseteq \mathfrak{a}^{2} M \subseteq N$. Since $N$ is weakly semiprime, $a_{i} M \subseteq N$. In particular, $a_{i} x_{i} \in N$. Hence $x=a_{1} x_{1}+\cdots+a_{n} x_{n} \in N$. This shows that $\mathfrak{a} M \subseteq N$.

The rest of the proof can be proved easily.

CoRollary 4.1. If every proper ideal of a ring $R$ is semiprime, then every proper submodule of a module over the ring $R$ is weakly semiprime.

Proof. Let $P$ be a proper submodule of a module $M$ over the ring $R$. Assume that $\mathfrak{a}$ is any ideal of $R$ with $\mathfrak{a}^{2} M \subseteq P$. Then $\mathfrak{a}^{2} \subseteq\left(P:_{R} M\right)$. Moreover, $\left(P:_{R} M\right)$ is a proper ideal of $R$, so by hypothesis $\left(P:_{R} M\right)$ is semiprime. Hence $\mathfrak{a} \subseteq\left(P:_{R} M\right)$, so that $\mathfrak{a} M \subseteq P$. By Lemma 4.1, $P$ is weakly semiprime.

If $P$ is a weakly semiprime submodule of an $R$-module $M$, then it is clear that $\left(P:_{R} M\right)$ is a proper radical ideal of $R$, that is, $\sqrt{\left(P:_{R} M\right)}=\left(P:_{R} M\right)$. It is known (see [9, Corollary 2.7] ) that, over a commutative ring whose proper ideals are semiprime, every proper submodule of a nonzero module is intersection of prime submodules.

Let $R$ be a Dedekind domain, and let $\mathfrak{a}$ be a nonzero proper ideal of $R$. It is well-known [2, Corollary 11.9] that $\mathfrak{a}$ is a finite product of (not necessarily distinct) prime ideals. Hence $\mathfrak{a}$ is semiprime if only if $\mathfrak{a}$ is a finite product of distinct prime ideals.

Definition 4.1. Let $P$ be a submodule of an $R$-module $M$. Then the residue class $R$-module $M / P$ is called a weakly semiprime module if $P$ is weakly semiprime.

Lemma 4.2. For each submodule $P$ of an $R$-module $M$, the following assertions are equivalent:

(a) The residue class $R$-module $M / P$ is weakly semiprime.

(b) For each ideal $\mathfrak{a}$ of $R$ with $\mathfrak{a}^{2}(M / P)=0, \mathfrak{a}(M / P)=0$.

(c) For each ideal $\mathfrak{a}$ of $R$ with $\mathfrak{a} M \subseteq\left(P:_{M} \mathfrak{a}\right), \mathfrak{a} M \subseteq P$.

(d) For each ideal $\mathfrak{a}$ of $R$ with $\mathfrak{a} M+\left(P:_{M} \mathfrak{a}\right)=\left(P:_{M} \mathfrak{a}\right), \mathfrak{a} M \subseteq P$.

(e) For each ideal $\mathfrak{a}$ of $R$ with $\mathfrak{a}\left(M /\left(P:_{M} \mathfrak{a}\right)\right)=0, M /\left(P:_{M} \mathfrak{a}\right)=0$.

An $R$-module $M$ is called meet irreducible or simply, irreducible if $M \neq 0$ and the intersection of two nonzero submodules of $M$ is always nonzero. A 
submodule $N$ of an $R$-module $M$ is called irreducible if the quotient $R$-module $M / N$ is irreducible. Let $M$ be an $R$-module. Then for any ideals $\mathfrak{a}, \mathfrak{b}$ of $R$ $(\mathfrak{a} \cap \mathfrak{b}) M \subseteq \mathfrak{a} M \cap \mathfrak{b} M$. However, the converse is not true in general.

If a submodule $N$ of an $R$-module $M$ is weakly semiprime, then by Lemma 4.1. $\left(N:_{R} M\right)$ is semiprime. In order $\left(N:_{R} M\right)$ to be prime, we need more conditions on $N$ as in the following results:

TheOREM 4.1. Let $M$ be a module over a ring $R$ such that for any ideals $\mathfrak{a}, \mathfrak{b}$ of $R,(\mathfrak{a} \cap \mathfrak{b}) M=\mathfrak{a} M \cap \mathfrak{b} M$. If $N$ is an irreducible and weakly semiprime submodule of $M$, then $\left(N:_{R} M\right)$ is a prime ideal of $R$.

Proof. Let us write $\mathfrak{p}=\left(N:_{R} M\right)$. Clearly, $\mathfrak{p} \neq R$. Suppose that $\mathfrak{p}$ is not prime. Then there exist $a, b \in R$ such that $a b \in \mathfrak{p}, a \notin \mathfrak{p}, b \notin \mathfrak{p}$. Then $a M \nsubseteq N$, so that there exists $m \in M$ such that $a m \notin N$. This implies that $a(m+N) \neq 0+N$ in $M / N$, so that $(\mathfrak{p}+R a)(M / N) \neq 0$ and by the same argument $(\mathfrak{p}+R b)(M / N) \neq 0$. Hence $(\mathfrak{p}+R a)(M / N) \cap(\mathfrak{p}+R b)(M / N) \neq 0$. However,

$$
((\mathfrak{p}+R a) \cap(\mathfrak{p}+R b))^{2}(M / N) \subseteq(\mathfrak{p}+R a)(\mathfrak{p}+R b)(M / N) \subseteq \mathfrak{p}(M / N)=0 .
$$

Since $N$ is weakly semiprime, it follows from Lemma 4.2 that

$$
((\mathfrak{p}+R a) \cap(\mathfrak{p}+R b))(M / N)=0,
$$

so that $(\mathfrak{p}+R a)(M / P) \cap(\mathfrak{p}+R b)(M / N)=0$. This contradiction shows that $\mathfrak{p}$ is prime.

Corollary 4.2. Let $R$ be a ring and $\mathfrak{p}$ be an ideal of $R$. If $\mathfrak{p}$ is an irreducible ideal of $R$ such that $z^{2} \in \mathfrak{p}(z \in R)$ implies $z \in \mathfrak{p}$, then $\mathfrak{p}$ is a prime ideal of $R$.

The following lemma is the statement just prior to [3, Theorem 1.6], but we include and prove here to use it in our classification and to get another result.

Lemma 4.3. If $M$ is a projective $R$-module, then

$$
\left(\cap_{\lambda \in \Lambda} \mathfrak{a}_{\lambda}\right) M=\cap_{\lambda \in \Lambda}\left(\mathfrak{a}_{\lambda} M\right)
$$

for any nonempy collection of ideals $\mathfrak{a}_{\lambda}(\lambda \in \Lambda)$ of $R$. 
Proof. Let $F$ be an $R$-free module and $N$ be a submodule of $F$ such that $F=M \oplus N$. It can be easily proven that $\left(\cap_{\lambda \in \Lambda} \mathfrak{a}_{\lambda}\right) F=\cap_{\lambda \in \Lambda}\left(\mathfrak{a}_{\lambda} F\right)$ for any nonempy collection of ideals $\mathfrak{a}_{\lambda}(\lambda \in \Lambda)$ of $R$. This implies that

$$
\begin{aligned}
\left(\cap_{\lambda \in \Lambda} \mathfrak{a}_{\lambda}\right) M \oplus\left(\cap_{\lambda \in \Lambda} \mathfrak{a}_{\lambda}\right) N & =\cap_{\lambda \in \Lambda}\left(\mathfrak{a}_{\lambda}(M \oplus N)\right) \\
& =\cap_{\lambda \in \Lambda}\left(\mathfrak{a}_{\lambda} M \oplus \mathfrak{a}_{\lambda} N\right) .
\end{aligned}
$$

It follows that $\left(\cap_{\lambda \in \Lambda} \mathfrak{a}_{\lambda}\right) M=\cap_{\lambda \in \Lambda}\left(\mathfrak{a}_{\lambda} M\right)$ and $\left(\cap_{\lambda \in \Lambda} \mathfrak{a}_{\lambda}\right) N=\cap_{\lambda \in \Lambda}\left(\mathfrak{a}_{\lambda} N\right)$. For otherwise, we can take

$$
x \in \cap_{\lambda \in \Lambda}\left(\mathfrak{a}_{\lambda} M\right) \backslash\left(\cap_{\lambda \in \Lambda} \mathfrak{a}_{\lambda}\right) M \quad \text { and } \quad y \in \cap_{\lambda \in \Lambda}\left(\mathfrak{a}_{\lambda} N\right) \backslash\left(\cap_{\lambda \in \Lambda} \mathfrak{a}_{\lambda}\right) N .
$$

Then by 4.1) $x+y \in\left(\cap_{\lambda \in \Lambda} \mathfrak{a}_{\lambda}\right) M \oplus\left(\cap_{\lambda \in \Lambda} \mathfrak{a}_{\lambda}\right) N=\cap_{\lambda \in \Lambda}\left(\mathfrak{a}_{\lambda} M \oplus \mathfrak{a}_{\lambda} N\right)$, so that for all $\lambda \in \Lambda$, there exist $u_{\lambda} \in \mathfrak{a}_{\lambda} M$ and $v_{\lambda} \in \mathfrak{a}_{\lambda} N$ such that $x+y=u_{\lambda}+v_{\lambda}$. Hence $x-u_{\lambda}=v_{\lambda}-y \in M \cap N=0$, so that $x=u_{\lambda}$ and $y=v_{\lambda}$. Hence $x \in \cap_{\lambda \in \Lambda}\left(\mathfrak{a}_{\lambda} M\right)$ and $y \in \cap_{\lambda \in \Lambda}\left(\mathfrak{a}_{\lambda} N\right)$. This is a contradiction.

If $M$ is a faithful multiplication module, then we can get the same result as in Lemma 4.3 (see [3, Theorem 1.6]).

Corollary 4.3. Let $M$ be a projective module or a faithful multiplication module over a ring $R$. If $N$ is an irreducible and weakly semiprime submodule of $M$, then $\left(N:_{R} M\right)$ is a prime ideal of $R$.

As a special case of Corollary 4.3 , Corollary 4.2 holds, as well. In other form, if $\mathfrak{p}$ is an irreducible ideal of a ring $R$ such that $\mathfrak{a}^{2} \subseteq \mathfrak{p}$ ( $\mathfrak{a}$ is an ideal of $R) \Rightarrow \mathfrak{a} \subseteq \mathfrak{p}$, then $\mathfrak{p}$ is a prime ideal of $R$.

\section{ACKNOWLEDGEMENTS}

The authors appreciate that the referee gave us some remarks, which made us much more logical reasoning toward the draft of the paper.

\section{REFERENCES}

[1] A. Barnard, Multiplication modules, J. Algebra 71 (1) (1981), 174-178.

[2] D. Eisenbud, "Commutative Algebra", Graduate Texts in Mathematics 150, Springer-Verlag, New York, 1995.

[3] Z. El-Bast, P.F. Smith, Multiplication modules, Comm. Algebra 16 (4) (1988), $755-779$. 
[4] J. Jenkins, P.F. Smith, On the prime radical of a module over a commutative ring, Comm. Algebra 20 (12) (1992), 3593-3602.

[5] S.T. Hu, "Introduction to Homological Algebra", Holden-Day, Inc., San Francisco-London-Amsterdam, 1968.

[6] T.Y. LAM, "Lectures on Modules and Rings", Graduate Texts in Mathematics 189, Springer-Verlag, New York, 1999.

[7] S.C. LEE, D.S. LEE, Direct sums of indecomposable injective modules, Bull. Austral. Math. Soc. 62 (1) (2000), 57-66.

[8] S.C. LeE, R. VArmazyar, Semiprime submodule of a module and related concepts, J. Algebra Appl. 18 (8) (2019), 1950147, 11 pp.

[9] R.L. McCasland, P.F. Smith, On isolated submodules, Comm. Algebra 34 (8) (2006), $2977-2988$.

[10] D.W. Sharpe, P. VÁmos, "Injective Modules", Cambridge Tracts in Mathematics and Mathematical Physics 62, Cambridge University Press, LondonNew York, 1972.

[11] H.A. TAvallaee, R. VARmazyar, Semi-radicals of submodules in modules, I. J. Engineering Science 19 (1) (2008), 21-27.

[12] F. WANG, H. KIM, Two generalizations of projective modules and their applications, J. Pure Appl. Algebra 219 (6) (2015), 2099-2123. 